\title{
Comparative Study between Intravenous and Intraperitoneal Magnesium Sulphate as Adjuvant to General Anesthesia for Pain Management in Laparoscopic Cholecystectomy
}

Mohamed Ahmed Mohamed Elfiky, Alsayed Mostafa Stohy, Wael Mohmed El Mahdy Ibrahim \&

Ahmed Khedr Tolba Ibrahim*

Department of Anesthesiology and Intensive Care, Faculty of Medicine, Al-Azhar University

*Corresponding Author: Ahmed Khedr Tolba Ibrahim, Email: dr.ahmedkhedr91@gmail.com

\begin{abstract}
Background: numerous researches have proved the benefits of laparoscopic cholecystectomy compared to the open method, mainly because of less metabolic response to stress, maintenance of diaphragm and pulmonary function, less postoperative complications, lower incidence of postoperative ileus, early mobilization, shorter hospital stay, and a more cosmetic. The main presentation of postoperative pain is somatic, whereas visceral pain is less present, and thus the pain is less in the patients operated by laparoscopic method. Aim of the Work: to compare intravenous Magnesium Sulphate with intra peritoneal Magnesium Sulphate as adjuvant to general anesthesia for pain management in laparoscopic cholecystectomy. Patients and Methods: this prospective randomized double blind clinical study using closed envelopes method was carried out on 100 adult patients, Undergoing laparoscopic cholecyctectomy in Bab-Alshaeria University Hospital, faculty of medicine, Al-Azhar University. After approval by the Institutional Ethical Committee, and informed written consent obtained from the patients, we randomly divided the patients into two groups 50 patients each. Results: the pain scores of group II (intra-peritoneal group) were significantly lower than group I (intravenous group), the total opioid consumption postoperatively in group II(intra-peritoneal group) was highly significant lower than group I (intravenous group). Also there was a significant reduction in opioid-related side effects such as postoperative nausea and vomiting. Conclusion: the intraperitoneal administration of magnesium sulphate is a safe and effective method in the management of acute postoperative pain after laparoscopic cholecystectomy more than intravenous administration of magnesium sulphate.
\end{abstract}

Keywords: Intravenous and Intraperitoneal Magnesium Sulphate - Anaestesia - Laparoscopic Cholecystectomy

\section{INTRODUCTION}

Laparoscopy has changed the surgical approach to symptomatic gallstone disease. The first laparoscopic cholecystectomy was performed in Europe in 1987 using a pneumoperitoneum. Many studies have shown the preference of laparoscopic cholecystectomy over open cholecystectomy, and laparoscopic cholecystectomy has become the standard procedure for gallstone disease and was first called the 'gold standard' in $1989^{(1)}$. However, pain is the most frequent complaint after LC in 17$41 \%$ of the patients and it is the main reason for staying overnight in the hospital on the day of the operation ${ }^{(1)}$. Consquently, Postoperative pain should be effectively treated. Effective treatment serves to blunt autonomic, somatic and endocrine reflexes with a resultant potential decrease in perioperative morbidity. The most common treatment practice is a poly pharmacological approach ${ }^{(2)}$. Pneumoperitoneum with carbon dioxide (CO2) insufflation for laparoscopic surgery induces a cardiovascular response characterized by elevations of arterial pressure and systemic vascular resistance with no significant change in heart rate. These vasopressor responses are likely to be due to increased release of catecholamines, vasopressin, or both. On the other hand, magnesium sulphate (MgSO4) blocks the release of catecholamines from both adrenergic nerve terminals and the adrenal gland, and intravenous (IV) magnesium sulphate inhibits catecholamine release associated with tracheal intubation. Moreover, magnesium produces vasodilatation by acting directly on blood vessels, and high-dose magnesium attenuates vasopressinstimulated vasoconstriction and normalizes sensitivity to vasopressin ${ }^{(3)}$. In addition, carbon dioxide reacts with the tissue and activates the emergence of sour products, which put local pressure on the nerve endings of the peritoneum and the right phrenic nerve ${ }^{(4)}$. Noxious stimulation leads to the release of glutamate and aspartate, which bind to various subclasses of excitatory amino acid receptors, including the $\mathrm{N}$-methyl D-aspartate (NMDA) receptor. Activation of NMDA receptors leads to calcium and sodium influx into the cell, with an efflux of potassium and initiation of central sensitization and windup. Magnesium blocks NMDA channels in a voltage-dependent way, and 
its addition produces a reduction of NMDA-induced currents ${ }^{(5)}$. It is well known that the peritoneum is slightly sensitive, free from pain on sharp, cutting and puncture wounds. Moreover, it is highly sensitive to distension, tearing and separation. Perioperatively, stronger pain occurs due to distension of the parietal peritoneum, and after the surgery pain in the right shoulder and the shoulder blade is evident due to irritation of phrenic nerve ${ }^{(6)}$. Studies related to magnesium sulfate (MgSO4) administration revealed that the anesthetic and analgesia quality may improve. The true site of action of magnesium is probably at the spinal cord NMDA receptors. Hence, it has been used as an adjunct to analgesics and anesthetic agents for intraoperative and postoperative analgesia. Several recent reports have described the efficacy of magnesium infusions in moderate dosage both during surgery and in the postoperative period for decreasing postoperative analgesic requirements ${ }^{(5)}$. In addition, it competes with calcium ions in synaptic junctions and prevents the release of presynaptic acetylcholine, prolonging the effects of neuromuscular blocker agents ${ }^{(7)}$.

\section{AIM OF THE WORK}

To compare intravenous Magnesium Sulphate with intra peritoneal Magnesium Sulphate as adjuvant to general anesthesia for pain management in laparoscopic cholecystectomy.

\section{PATIENTS AND METHODS}

This prospective randomized double blind clinical study using closed envelopes method was carried out on 100 adult patients, Undergoing laparoscopic cholecyctectomy in Bab-Alshaeria university hospital, faculty of medicine, Al-Azhar University. After approval by the Institutional Ethical Committee, and informed written consent obtained from the patients, we randomly divided the patients into two groups 50 patients each. Patients were prepared by 8 hours preoperative fasting, receiving tablet Alprazolam $0.25 \mathrm{mg}$ and Omeprazole $20 \mathrm{mg}$ at bed time day before surgery and morning of surgery. All patients were educated about the standard Visual Analogue Scale (VAS) pain score of 0-10, during pre anaesthetic evaluation visit. Groups: Group1: was received Magnesium sulphate $50 \mathrm{mg} / \mathrm{kg}$ in $250 \mathrm{ml}$ of isotonic $0.9 \%$ N.S intravenously over 30 minutes with the beginning of operation. Group2: was received Magnesium sulphate $50 \mathrm{mg} / \mathrm{kg}$ in $30 \mathrm{ml}$ of isotonic $0.9 \%$ N.S intra peritoneal at the end of surgery. Inclusion criteria: 1- ASA physical status I-II. 2- Both genders are eligible for study. 3Patients age 18to 60 years. 4- Patients undergoing laparoscopic cholecystectomy. Exclusion Criteria: 1- ASA III, IV and V class patients. 2- Presence of psychiatric disease. 3- Obese patients (BMI $>30 \mathrm{~kg}$ m2). 4- Heart block. 5- Renal impairment. 6Severe chronic disease. 7- Those with an allergy to any of the study drugs were excluded from the study. 8- Patients with previous heart surgery, left ventricular ejection fraction of less than $40 \%$, documented myocardial infarction within the previous six weeks, congestive heart failure, severe chronic obstructive pulmonary disease and/or history of steroid or bronchodilator consumption, renal insufficiency (creatinine $>2 \mathrm{mg} / \mathrm{dL}$ ), liver disease. 9- Patients treated with calcium channel blockers and pregnancy. 10- Patients with increased risk of regurgitation. Preoperative assessment: Routine preoperative assessment was carried out to fulfill patient's criteria for the study by full history taking, physical examination including chest and heart examination as well as reviewing the patient's investigations $(\mathrm{CBC}, \mathrm{S}$. creatinine, blood urea, SGOT, SGPT, PT, PTT, INR, ECG, and chest X-Ray). Study protocol was explained to the patients taking their consent. No premedication was prescribed on the morning of surgery. Materials and Equipments: Equipments for GA: IV line, I.V fluids, suction apparatus, airways, laryngoscope with different size blades, endotracheal tubes of variable sizes, electrical cardioversion (DC) and equipments for difficult intubation. Drugs for the technique: Normal Saline 0.9\%, Magnesium sulphate. Drugs for GA: Propofol, atracurium, Fentanyl, Isoflurane, Atropine and Neostigmine. Monitoring: In the operating room, monitoring of ECG, heart rate (HR), oxygen saturation and noninvasive blood pressure was started. After sterilization an intravenous line was secured with an 18-gauge cannula and all patients received an infusion of $0.9 \%$ saline $5 \mathrm{ml} / \mathrm{kg}$ before the start of the study. The patients were allocated randomly to one of two groups using a computer-generated list. The intravenous group $(n=50)$ received Magnesium sulphate $50 \mathrm{mg} / \mathrm{kg}$ in $250 \mathrm{ml}$ of isotonic $0.9 \%$ N.S intravenously over 30 minutes with the beginning of operation. The intraperitoneal group $(n=50)$ received Magnesium sulphate $50 \mathrm{mg} / \mathrm{kg}$ in $30 \mathrm{ml}$ of 
isotonic $0.9 \%$ N.S intra peritoneal at the end of surgery. Induction: During the administration of the preoperative medication patients pulse, blood pressure, and oxygen saturation were monitored. After this a Ringer lactate infusion at rate of $10 \mathrm{ml}$ $/ \mathrm{kg}$ was started. Fentanyl 1. $\mu \mathrm{g} / \mathrm{kg}$ was given 5 minutes before induction. After 3 minutes of pre oxygenation, anesthesia was induced with Propofol $2.0 \mathrm{mg} / \mathrm{kg}$ body weight over 30 seconds and injection Atracurium $0.5 \mathrm{mg} / \mathrm{kg}$ body weight, Patients were maintained with $50 \%$ O2, 1.2 vol\% isoflurane, Laryngoscopy was performed $60 \mathrm{~s}$ after the administration of propofol using an appropriate size of Macintosh blade. Cuffed tracheal tubes of internal diameter 7 and $7.5 \mathrm{~mm}$ were used in female and male patients, respectively. The cuff of the tracheal tube was inflated gently to the minimum pressure required to prevent a gas leak on positive pressure ventilation. After anesthesia induction, pneumoperitoneum was created by insufflation of $\mathrm{CO} 2$ to maintain intra-abdominal pressure between 12 and $15 \mathrm{mmHg}$ throughout the surgical procedure. Maintenance: After tracheal intubation and evaluation of intubation condition, anesthesia was maintained with $1.2 \%$ Isoflurane in $\mathrm{O} 2$ via a closed circuit system and neuromuscular blockade was maintained with atracurium $0.1-0.2 \mathrm{mg} / \mathrm{kg}$ as on demand. Mechanical ventilation was provided by Dragger anesthesia machine and the respiratory rate and tidal volume were adjusted to maintain the end-tidal $\mathrm{CO} 2$ around $35 \mathrm{mmHg}$. Recovery: At the end of surgery, inhalational anesthesia was stopped, then the residual neuro-muscular block was reversed with Neostigmine $(0.05 \mathrm{mg} / \mathrm{kg})$ and Atropine $(0.02 \mathrm{mg} / \mathrm{kg})$ and when the patient fulfilled extubation criteria the endotracheal tube was withdrawn and patients transferred to the PACU. Intraoperative measurements: The following measures were assessed and recorded: Arterial pressure and heart rate, measured before induction (baseline), after intubation (Tin), before pneumoperitoneum ( $\mathrm{P} 0$ ), every $10 \mathrm{~min}$ after pneumoperitoneum for $30 \mathrm{~min}$ (P10, P20, and P30), after extubation (Tex), and before discharge from the operating room. Surgical duration, corresponding to the time from skin incision until skin closure. Time of emergence (time to first response to a simple verbal command following discontinuation of anesthesia).
In the post anaesthesia care unit (PACU): Modified Aldrete Score evaluated in the PACU every 5 min until discharge. Patients were ready for discharge upon achieving an Aldrete score of 9 or higher. Time to achieve an Aldrete score of at least 9 corresponded to the time from arrival at the PACU until discharge to the intermediate care unit. In the ward: Patient evaluated regarding to incisional, intra-abdominal, and shoulder pain at the first, second, third, sixth, and 24th postoperative hour using VAS. When the VAS score was 4 or higher, patients were given 50 $\mathrm{mg}$ intramuscular pethedine. Visual analogue scale (VAS): The patient was simply instructed and asked to correlate the degree of his pain on a scale for pain assessment graded from 0 to 10 ( 0 as "no pain" to 10 as "worst imaginable pain"). To indicate how much pain they are currently feeling. The far left end (0) indicates 'No pain' and the far right end (10) indicates 'Worst pain ever'. The time to first analgesic administration, number of analgesic requests in the first $24 \mathrm{~h}$, and total analgesic requirement during the first postoperative $24 \mathrm{~h}$. The occurrence of any adverse events, including hypoventilation (bradypnea respiratory rate (RR) $<10 \mathrm{bpm}), \mathrm{SpO} 2$ reaching $92 \%$ or less, sedation, hypotension (mean arterial pressure (MAP) $<55 \mathrm{mmHg}$ ), bradycardia (heart rate (HR) $<60 \mathrm{bpm}$ ), nausea, and vomiting. Nausea was assessed at the same intervals of VAS using a scoring system $(0=$ none, $1=$ mild, $2=$ moderate, $3=$ severe). Patients suffering from vomiting or who rated their nausea at level 2 or more received 4 $\mathrm{mg}$ intravenous ondansetron and their postoperative antiemetic needs were recorded. Sedation was also assessed at the same intervals of VAS using a four-point scale $(0=$ alert, $1=$ quietly awake, 2 = asleep but easily aroused, and $3=$ deep sleep). Statistical analysis: Recorded data were analyzed using the statistical package for social sciences, version 20.0 (SPSS Inc., Chicago, Illinois, USA). Quantitative data were expressed as mean \pm standard deviation (SD). Qualitative data were expressed as frequency and percentage. 


\section{RESULTS}

Table (1): Comparison between groups according to demographic data

\begin{tabular}{|l|c|c|c|c|}
\hline $\begin{array}{c}\text { Demographic } \\
\text { Data }\end{array}$ & $\begin{array}{c}\text { Group I: IV } \\
\text { (N=50) }\end{array}$ & $\begin{array}{c}\text { Group II: } \\
\text { Intraperitoneal } \\
(\mathbf{N}=50)\end{array}$ & t/x2\# & $\begin{array}{c}\mathbf{p}- \\
\text { value }\end{array}$ \\
\hline $\begin{array}{l}\text { Age(years) } \\
\text { Mean+SD } \\
\text { Range }\end{array}$ & $\begin{array}{c}38.16 \pm 11.48 \\
19-60\end{array}$ & $\begin{array}{c}39.18 \pm 10.91 \\
19-60\end{array}$ & 0.207 & 0.650 \\
\hline Sex & $39(78.0 \%)$ & $40(80.0 \%)$ & $0.060 \#$ & 0.806 \\
Female & $11(22.0 \%)$ & $10(20.0 \%)$ & & \\
Male & $39(78.0 \%)$ & $36(72.0 \%)$ & $0.480 \#$ & 0.488 \\
\hline ASA & $11(22.0 \%)$ & $14(28.0 \%)$ & \\
I & & &
\end{tabular}

t- Independent Sample t-test; $x^{2}$ : Chi-square test; $p$-value $>0.05$ NS

This table shows no statistically significant difference between the groups according to demographic data.

Table (2): Comparison between groups according to SBP

\begin{tabular}{|l|c|c|c|c|}
\hline $\begin{array}{l}\text { SBP } \\
(\mathbf{m m H g})\end{array}$ & $\begin{array}{c}\text { Group I: IV } \\
\text { (N=50) }\end{array}$ & $\begin{array}{c}\text { Group II: } \\
\text { Intraperitoneal } \\
\text { (N=50) }\end{array}$ & t-test & p-value \\
\hline $\begin{array}{l}\text { Baseline } \\
\text { Mean+SD } \\
\text { Range }\end{array}$ & $\begin{array}{c}122.16 \pm 11.65 \\
108-156\end{array}$ & $\begin{array}{c}128.58 \pm 13.12 \\
110-167\end{array}$ & 3.694 & $0.011^{*}$ \\
\hline $\begin{array}{l}\text { Tin } \\
\text { Mean+SD } \\
\text { Range }\end{array}$ & $\begin{array}{c}115.96 \pm 10.99 \\
84-143\end{array}$ & $\begin{array}{c}125.56 \pm 16.25 \\
103-180\end{array}$ & 3.979 & $0.021^{*}$ \\
\hline $\begin{array}{l}\text { P0 } \\
\text { Mean+SD } \\
\text { Range }\end{array}$ & $\begin{array}{c}110.50 \pm 14.76 \\
83-151\end{array}$ & $\begin{array}{c}117.18 \pm 11.26 \\
91-141\end{array}$ & 4.485 & $0.019^{*}$ \\
\hline $\begin{array}{l}\text { P10 } \\
\text { Mean+SD } \\
\text { Range }\end{array}$ & $\begin{array}{c}114.46 \pm 10.34 \\
93-145\end{array}$ & $\begin{array}{c}120.08 \pm 10.82 \\
102-152\end{array}$ & 7.053 & $0.009^{*}$ \\
\hline $\begin{array}{l}\text { P20 } \\
\text { Mean+SD } \\
\text { Range }\end{array}$ & $\begin{array}{c}112.44 \pm 8.79 \\
95-146\end{array}$ & $\begin{array}{c}117.76 \pm 18.64 \\
12-143\end{array}$ & 3.323 & $0.034^{*}$ \\
\hline $\begin{array}{l}\text { P30 } \\
\text { Mean } \pm S D \\
\text { Range }\end{array}$ & $\begin{array}{c}120.45 \pm 11.32 \\
100-156\end{array}$ & $121.38 \pm 9.83$ & 0.182 & 0.670 \\
\hline $\begin{array}{l}\text { Tex } \\
\text { Mean+SD } \\
\text { Range }\end{array}$ & $\begin{array}{c}126.24 \pm 12.39 \\
100-157\end{array}$ & $139.62 \pm 12.69$ \\
\hline $\begin{array}{l}\text { Before } \\
\text { discharge } \\
\text { Mean+SD } \\
\text { Range }\end{array}$ & $\begin{array}{c}1170-171 \\
107-144\end{array}$ & 28.465 & $<0.001^{* *}$ \\
\hline
\end{tabular}

t- Independent Sample t-test; *p-value $<0.05 \mathrm{~S}$; **p-value $<0.001$ HS \& p-value $>0.05 \mathrm{NS}$

This table indicates statistically significant difference between the groups according to SBP from baseline to before discharge.
Table (3): Comparison between groups according to DBP

\begin{tabular}{|c|c|c|c|c|}
\hline$\underset{(\mathbf{m m H g})}{\text { DBP }}$ & $\begin{array}{c}\text { Group I: IV } \\
(\mathbf{N}=\mathbf{5 0})\end{array}$ & $\begin{array}{c}\text { Group II: } \\
\text { Intraperitoneal } \\
(\mathrm{N}=\mathbf{5 0})\end{array}$ & t-test & p-value \\
\hline $\begin{array}{l}\text { Baseline } \\
\text { Mean } \pm \text { SD } \\
\text { Range } \\
\end{array}$ & $\begin{array}{c}73.74 \pm 6.71 \\
56-90\end{array}$ & $\begin{array}{c}78.66 \pm 8.31 \\
68-99\end{array}$ & 10.608 & $0.002^{*}$ \\
\hline $\begin{array}{l}\text { Tin } \\
\text { Mean } \pm \text { SD } \\
\text { Range }\end{array}$ & $\begin{array}{c}73.24 \pm 10.30 \\
40-97\end{array}$ & $\begin{array}{c}76.92 \pm 8.87 \\
54-93\end{array}$ & 2.125 & $0.024 *$ \\
\hline $\begin{array}{l}\text { P0 } \\
\text { Mean } \pm \text { SD } \\
\text { Range }\end{array}$ & $\begin{array}{c}70.54 \pm 10.87 \\
45-96\end{array}$ & $\begin{array}{c}74.50 \pm 9.73 \\
47-87\end{array}$ & 3.216 & $0.013^{*}$ \\
\hline $\begin{array}{l}\text { P10 } \\
\text { Mean } \pm \text { SD } \\
\text { Range }\end{array}$ & $\begin{array}{c}67.08 \pm 7.81 \\
46-86\end{array}$ & $\begin{array}{c}72.34 \pm 7.56 \\
54-91 \\
\end{array}$ & 4.498 & $0.036^{*}$ \\
\hline $\begin{array}{l}\text { P20 } \\
\text { Mean } \pm \text { SD } \\
\text { Range }\end{array}$ & $\begin{array}{c}71.86 \pm 9.11 \\
50-93\end{array}$ & $\begin{array}{c}73.40 \pm 8.53 \\
52-91\end{array}$ & 2.976 & $0.035^{*}$ \\
\hline $\begin{array}{l}\text { P30 } \\
\text { Mean } \pm \text { SD } \\
\text { Range } \\
\end{array}$ & $\begin{array}{c}72.91 \pm 8.37 \\
57-91\end{array}$ & $\begin{array}{c}71.48 \pm 8.01 \\
49-85\end{array}$ & 0.730 & 0.395 \\
\hline $\begin{array}{l}\text { Tex } \\
\text { Mean } \pm \text { SD } \\
\text { Range }\end{array}$ & $\begin{array}{c}77.10 \pm 8.68 \\
56-93\end{array}$ & $\begin{array}{c}84.42 \pm 7.62 \\
64-97\end{array}$ & 20.065 & $<0.001$ *** \\
\hline $\begin{array}{l}\text { Before } \\
\text { discharge } \\
\text { Mean } \pm \text { SD } \\
\text { Range }\end{array}$ & $\begin{array}{c}71.36 \pm 7.12 \\
60-91\end{array}$ & $\begin{array}{c}74.78 \pm 8.89 \\
62-107\end{array}$ & 3.902 & $0.038^{*}$ \\
\hline
\end{tabular}

t- Independent Sample t-test; *p-value $<0.05 \mathrm{~S}$; **p-value $<0.001$ HS \& p-value $>0.05$ NS

This table shows statistically significant difference between groups according to DBP from baseline to before discharge.

Table (4): Comparison between groups according to HR

\begin{tabular}{|c|c|c|c|c|}
\hline HR (b/m) & $\underset{(\mathbf{N}=\mathbf{5 0})}{\text { Group I: IV }}$ & $\begin{array}{c}\text { Group II: } \\
\text { Intraperitoneal } \\
(\mathbf{N}=\mathbf{5 0})\end{array}$ & t-test & $\begin{array}{c}\text { p- } \\
\text { value }\end{array}$ \\
\hline $\begin{array}{l}\text { Baseline } \\
\text { Mean } \pm S D \\
\text { Range }\end{array}$ & $\begin{array}{c}85.86 \pm 12.59 \\
65-117\end{array}$ & $\begin{array}{l}93.20 \pm 14.06 \\
53-123\end{array}$ & 7.563 & $0.007^{*}$ \\
\hline $\begin{array}{l}\text { T in } \\
\text { Mean } \pm \text { SD } \\
\text { Range }\end{array}$ & $\begin{array}{c}89.82 \pm 10.97 \\
51-109\end{array}$ & $\begin{array}{c}105.96 \pm 99.29 \\
65-788\end{array}$ & 3.305 & $0.026^{*}$ \\
\hline $\begin{array}{l}\text { P0 } \\
\text { Mean } \pm \text { SD } \\
\text { Range }\end{array}$ & $\begin{array}{c}81.24 \pm 9.74 \\
50-107\end{array}$ & $\begin{array}{c}84.84 \pm 13.14 \\
63-139\end{array}$ & 3.421 & $0.023^{*}$ \\
\hline $\begin{array}{l}\text { P10 } \\
\text { Mean } \pm \text { SD } \\
\text { Range }\end{array}$ & $\begin{array}{c}78.72 \pm 9.45 \\
53-100\end{array}$ & $\begin{array}{l}82.28 \pm 10.25 \\
64-110\end{array}$ & 4.695 & $0.013^{*}$ \\
\hline $\begin{array}{l}\text { P20 } \\
\text { Mean } \pm \text { SD } \\
\text { Range }\end{array}$ & $\begin{array}{c}77.56 \pm 13.86 \\
60-95\end{array}$ & $\begin{array}{l}80.22 \pm 8.23 \\
62-98\end{array}$ & 2.997 & $0.047^{*}$ \\
\hline $\begin{array}{l}\text { P30 } \\
\text { Mean } \pm \text { SD } \\
\text { Range }\end{array}$ & $\begin{array}{c}80.06 \pm 9.97 \\
57-103 \\
\end{array}$ & $\begin{array}{c}83.08 \pm 10.31 \\
50-110\end{array}$ & 3.222 & $0.039 *$ \\
\hline $\begin{array}{l}\text { Tex } \\
\text { Mean } \pm \text { SD } \\
\text { Range }\end{array}$ & $\begin{array}{c}92.14 \pm 12.29 \\
53-119\end{array}$ & $\begin{array}{c}96.28 \pm 13.31 \\
73-136\end{array}$ & 2.896 & $0.031 *$ \\
\hline $\begin{array}{l}\text { Before } \\
\text { discharge } \\
\text { Mean } \pm \text { SD } \\
\text { Range }\end{array}$ & $\begin{array}{c}80.80 \pm 8.50 \\
57-105\end{array}$ & $\begin{array}{c}77.94 \pm 6.36 \\
65-96\end{array}$ & 3.824 & $0.022 *$ \\
\hline
\end{tabular}

t- Independent Sample t-test; *p-value $<0.05 \mathrm{~S}$ 
This table demonstrates statistically significant difference between groups according to HR from baseline to before discharge.

Table (5): Comparison between groups according to surgical duration (min)

\begin{tabular}{|l|c|c|c|c|}
\hline $\begin{array}{c}\text { Surgical } \\
\text { duration } \\
\text { (min) }\end{array}$ & $\begin{array}{c}\text { Group I: } \\
\text { IV (N=50) }\end{array}$ & $\begin{array}{c}\text { Group II: } \\
\text { Intraperitoneal } \\
\text { (N=50) }\end{array}$ & t-test & $\begin{array}{c}\text { p- } \\
\text { value }\end{array}$ \\
\cline { 1 - 3 } Mean \pm SD & $38.48 \pm 8.35$ & $36.38 \pm 6.84$ & \multirow{2}{*}{1.892} & 0.172 \\
\hline Range & $23-55$ & $20-50$ & & \\
\hline
\end{tabular}

t- Independent Sample t-test; p-value >0.05 NS

This table displays no statistically significant difference between groups according to surgical duration (min).

Table (6): Comparison between groups according to time of emergence ( $\mathrm{min}$ )

\begin{tabular}{|c|c|c|c|c|}
\hline $\begin{array}{c}\text { Time of } \\
\text { emergence } \\
(\mathbf{m i n})\end{array}$ & $\begin{array}{c}\text { Group I: } \\
\text { IV }(\mathbf{N}=\mathbf{5 0})\end{array}$ & $\begin{array}{c}\text { Group II: } \\
\text { Intraperitoneal } \\
(\mathbf{N}=\mathbf{5 0})\end{array}$ & t-test & p-value \\
\hline Mean \pm SD & $14.46 \pm 2.31$ & $9.08 \pm 3.53$ & 8.501 & $<0.001 * *$ \\
\hline Range & $10-20$ & $3-20$ & & \\
\hline
\end{tabular}

t- Independent Sample t-test; **p-value <0.001 HS

This table shows highly statistically significant difference between the groups according to time of emergence (min).

Table (7): Comparison between groups according to modified alderet score

\begin{tabular}{|c|c|c|c|c|}
\hline $\begin{array}{l}\text { Modified } \\
\text { alderet } \\
\text { score }\end{array}$ & $\begin{array}{l}\text { Group I: } \\
\text { IV }(\mathrm{N}=\mathbf{5 0})\end{array}$ & $\begin{array}{c}\text { Group II: } \\
\text { Intraperitoneal } \\
(\mathbf{N}=\mathbf{5 0})\end{array}$ & z-test & p-value \\
\hline $\begin{array}{l}\mathbf{m i n} \\
\text { Mean }+ \text { SD } \\
\text { Range } \\
\end{array}$ & $\begin{array}{c}7.04 \pm 0.81 \\
6-9\end{array}$ & $\begin{array}{c}8.96 \pm 0.64 \\
8-10\end{array}$ & 17.222 & $<0.001^{* *}$ \\
\hline $\begin{array}{l}\mathbf{1 0} \mathbf{m i n} \\
\text { Mean } \pm \text { SD } \\
\text { Range }\end{array}$ & $\begin{array}{c}8.42 \pm 0.88 \\
7-10\end{array}$ & $\begin{array}{c}9.76 \pm 0.43 \\
9-10\end{array}$ & 9.007 & $<0.001^{* *}$ \\
\hline $\begin{array}{l}15 \mathrm{~min} \\
\text { Mean } \pm \text { SD } \\
\text { Range }\end{array}$ & $\begin{array}{c}9.26 \pm 0.61 \\
8-10\end{array}$ & $\begin{array}{c}10.00 \pm 0.00 \\
10-10\end{array}$ & 4.785 & $0.012 *$ \\
\hline $\begin{array}{l}20 \text { min } \\
\text { Mean } \pm \text { SD } \\
\text { Range }\end{array}$ & $\begin{array}{c}9.88 \pm 0.39 \\
9-10\end{array}$ & $\begin{array}{c}10.00 \pm 0.00 \\
10-10\end{array}$ & 1.756 & 0.440 \\
\hline $\begin{array}{l}\mathbf{2 5} \mathbf{m i n} \\
\text { Mean } \pm \text { SD } \\
\text { Range }\end{array}$ & $\begin{array}{c}9.80 \pm 1.41 \\
0-10\end{array}$ & $\begin{array}{c}10.00 \pm 0.00 \\
10-10\end{array}$ & 1.000 & 0.320 \\
\hline $\begin{array}{l}\mathbf{3 0} \mathbf{m i n} \\
\text { Mean } \pm \text { SD } \\
\text { Range }\end{array}$ & $\begin{array}{c}10.00 \pm 0.00 \\
10-10\end{array}$ & $\begin{array}{c}10.00 \pm 0.00 \\
10-10\end{array}$ & 0.000 & 1.000 \\
\hline
\end{tabular}

z- Mann-Whitney t-test; *p-value $<0.05 \mathrm{~S}$; **p-value $<0.001$ HS \& p-value $>0.05 \mathrm{NS}$
This table reveals statistically significant difference between the groups according to modified alderet score at $5 \mathrm{~min}, 10 \mathrm{~min}$ and $15 \mathrm{~min}$.

Table (8): Comparison between groups according to vAS incisional pain

\begin{tabular}{|c|c|c|c|c|}
\hline $\begin{array}{c}\text { VAS } \\
\text { Incisional } \\
\text { pain } \\
\end{array}$ & $\begin{array}{c}\text { Group I: } \\
\text { IV } \\
(\mathbf{N}=\mathbf{5 0})\end{array}$ & $\begin{array}{c}\text { Group II: } \\
\text { Intraperitoneal } \\
(\mathbf{N}=\mathbf{5 0})\end{array}$ & z-test & p-value \\
\hline $\begin{array}{l}\mathbf{1 s t} \mathbf{h r} \\
\text { Mean } \pm \text { SD } \\
\text { Range } \\
\end{array}$ & $\begin{array}{c}1.78 \pm 2.79 \\
0-8\end{array}$ & $\begin{array}{c}0.22 \pm 0.82 \\
0-5\end{array}$ & 14.361 & $<0.001 * *$ \\
\hline $\begin{array}{l}\text { 2nd hr } \\
\text { Mean } \pm \text { SD } \\
\text { Range }\end{array}$ & $\begin{array}{c}1.42 \pm 2.16 \\
0-7\end{array}$ & $\begin{array}{c}0.94 \pm 1.54 \\
0-7\end{array}$ & 3.636 & $0.039^{*}$ \\
\hline $\begin{array}{l}\text { 3rd hr } \\
\text { Mean } \pm \text { SD } \\
\text { Range } \\
\end{array}$ & $\begin{array}{c}1.58 \pm 2.50 \\
0-7\end{array}$ & $\begin{array}{c}1.16 \pm 1.91 \\
0-6\end{array}$ & 5.891 & $0.023^{*}$ \\
\hline $\begin{array}{l}\text { 6th hr } \\
\text { Mean } \pm \text { SD } \\
\text { Range } \\
\end{array}$ & $\begin{array}{c}1.76 \pm 2.35 \\
0-7\end{array}$ & $\begin{array}{c}2.32 \pm 2.55 \\
0-7 \\
\end{array}$ & 4.302 & $0.026^{*}$ \\
\hline $\begin{array}{l}\text { 24th hr } \\
\text { Mean } \pm \text { SD } \\
\text { Range }\end{array}$ & $\begin{array}{c}0.14 \pm 0.70 \\
0-4\end{array}$ & $\begin{array}{c}0.80 \pm 1.82 \\
0-7\end{array}$ & 5.737 & $0.019^{*}$ \\
\hline
\end{tabular}

z- Mann-Whitney t-test; *p-value $<0.05 \mathrm{~S}$; $* *$ p-value $<0.001$ HS

This table spointes statistically significant difference between groups according to VAS incisional pain from $1^{\text {st }} \mathrm{hr}$ to $24^{\text {th }} \mathrm{hr}$.

Table (9): Comparison between groups according to intra-bdominal pain

\begin{tabular}{|l|c|c|c|c|}
\hline $\begin{array}{c}\text { Intra- } \\
\text { abdominal } \\
\text { pain: }\end{array}$ & $\begin{array}{c}\text { Group I: } \\
\text { IV } \\
\text { (N=50) }\end{array}$ & $\begin{array}{c}\text { Group II: } \\
\text { Intraperitoneal } \\
\text { (N=50) }\end{array}$ & t-test & p-value \\
\hline $\begin{array}{l}\text { 1st hr } \\
\text { Mean } \pm S D \\
\text { Range }\end{array}$ & $\begin{array}{c}2.04 \pm 2.36 \\
0-8\end{array}$ & $\begin{array}{c}0.32 \pm 0.79 \\
0-3\end{array}$ & 23.780 & $<0.001^{* *}$ \\
\hline $\begin{array}{l}\text { 2nd hr } \\
\text { Mean } \pm S D \\
\text { Range }\end{array}$ & $\begin{array}{c}1.34 \pm 1.87 \\
0-5\end{array}$ & $\begin{array}{c}0.64 \pm 1.19 \\
0-5\end{array}$ & 4.987 & $0.028^{*}$ \\
\hline $\begin{array}{l}\text { 3rd hr } \\
\text { Mean } \pm S D \\
\text { Range }\end{array}$ & $\begin{array}{c}0.98 \pm 1.67 \\
0-5\end{array}$ & $\begin{array}{c}0.64 \pm 1.71 \\
0-7\end{array}$ & 4.010 & $0.017^{*}$ \\
\hline $\begin{array}{l}\text { 6th hr } \\
\text { Mean } \pm S D \\
\text { Range }\end{array}$ & $\begin{array}{c}1.42 \pm 2.09 \\
0-7\end{array}$ & $\begin{array}{c}0.70 \pm 1.63 \\
0-7\end{array}$ & 3.685 & $0.038^{*}$ \\
\hline $\begin{array}{l}\text { 24th hr } \\
\text { Mean } \pm S D \\
\text { Range }\end{array}$ & $\begin{array}{c}0.08 \pm 0.40 \\
0-2\end{array}$ & $0.12 \pm 0.63$ & 4.540 & $0.038^{*}$ \\
\hline
\end{tabular}

t- Independent Sample t-test; *p-value <0.05 S; **p-value $<0.001 \mathrm{HS}$

This table elucidates statistically significant difference between the groups according to intraabdominal pain from $1^{\text {st }} \mathrm{hr}$ to $24^{\text {th }} \mathrm{hr}$. 
Table (10): Comparison between the groups according to shoulder pain

\begin{tabular}{|l|c|c|c|c|}
\hline $\begin{array}{l}\text { Shoulder } \\
\text { pain: }\end{array}$ & $\begin{array}{c}\text { Group I: } \\
\text { IV } \\
(\mathbf{N}=\mathbf{5 0})\end{array}$ & $\begin{array}{c}\text { Group II: } \\
\text { Intraperitoneal } \\
(\mathbf{N}=\mathbf{5 0})\end{array}$ & t-test & p-value \\
\hline $\begin{array}{l}\text { 1st hr } \\
\text { Mean } \pm \text { SD } \\
\text { Range }\end{array}$ & $\begin{array}{c}1.10 \pm 2.10 \\
0-7\end{array}$ & $\begin{array}{c}0.10 \pm 0.51 \\
0-3\end{array}$ & 10.699 & $<0.001^{* *}$ \\
\hline $\begin{array}{l}\mathbf{2}^{\text {nd }} \mathbf{h r} \\
\text { Mean } \pm \text { SD } \\
\text { Range }\end{array}$ & $\begin{array}{c}1.46 \pm 2.15 \\
0-7\end{array}$ & $\begin{array}{c}0.00 \pm 0.00 \\
0-0\end{array}$ & 22.599 & $<0.001^{* *}$ \\
\hline $\begin{array}{l}\text { 3rd hr } \\
\text { Mean } \pm S D \\
\text { Range }\end{array}$ & $\begin{array}{c}1.40 \pm 2.29 \\
0-7\end{array}$ & $0.06 \pm 0.42$ \\
\hline $\begin{array}{l}\text { 6th hr } \\
\text { Mean } \pm S D \\
\text { Range }\end{array}$ & $\begin{array}{c}2.66 \pm 2.44 \\
0-7\end{array}$ & $0.39 \pm 1.08$ & 16.488 & $<0.001^{* *}$ \\
\hline $\begin{array}{l}\text { 24th hr } \\
\text { Mean } \pm S D \\
\text { Range }\end{array}$ & $\begin{array}{c}0.60 \pm 1.46 \\
0-6\end{array}$ & $0.06 \pm 0.42$ & 35.733 & $<0.001^{* *}$ \\
\hline
\end{tabular}

t- Independent Sample t-test; *p-value $<0.05 \mathrm{~S}$; **p-value $<0.001 \mathrm{HS}$

This table shows statistically significant difference between groups according to shoulder pain from $1^{\text {st }}$ hr to $24^{\text {th }} \mathrm{hr}$.

Table (11): Comparison between groups according to time to first analgesic administration

\begin{tabular}{|l|c|c|c|c|}
\hline $\begin{array}{c}\text { Time to first } \\
\text { analgesic } \\
\text { administration }\end{array}$ & $\begin{array}{c}\text { Group I: } \\
\text { IV } \\
\text { (N=50) }\end{array}$ & $\begin{array}{c}\text { Group II: } \\
\text { Intraperitoneal } \\
\text { (N=50) }\end{array}$ & t-test & p-value \\
\hline Mean \pm SD & $1.82 \pm 1.60$ & $5.00 \pm 2.38$ & \multirow{2}{*}{58.947} & $<0.001^{* *}$ \\
\hline Range & $0-10$ & $1-10$ & \\
\hline
\end{tabular}

t- Independent Sample t-test; **p-value <0.001 HS

This table presents highly statistically significant difference between the groups according to time to first analgesic administration.

Table (12): Comparison between groups according to number of analgesic requests in the first $24 \mathrm{~h}$

\begin{tabular}{|c|c|c|c|c|}
\hline $\begin{array}{c}\text { Number } \\
\text { of } \\
\text { analgesic } \\
\text { requests } \\
\text { in the } \\
\text { first 24h }\end{array}$ & $\begin{array}{c}\text { Group I: } \\
\text { IV } \\
(\mathbf{N}=\mathbf{5 0})\end{array}$ & $\begin{array}{c}\text { Group II: } \\
\text { Intraperitoneal } \\
\text { (N=50) }\end{array}$ & t-test & p-value \\
\cline { 1 - 3 } MeanD & $2.64 \pm 1.08$ & $1.40 \pm 0.88$ & 39.438 & $<0.001^{* *}$ \\
\hline Range & $0-5$ & $0-3$ & \\
\hline
\end{tabular}

t- Independent Sample t-test; **p-value <0.001 HS

This table shows highly statistically significant difference between groups according to number of analgesic requests in the first $24 \mathrm{~h}$.
Table (13): Comparison between groups according to total analgesic requirement during the first postoperative $24 \mathrm{~h}$

\begin{tabular}{|c|c|c|c|c|}
\hline $\begin{array}{c}\text { Total } \\
\text { analgesic } \\
\text { requirement } \\
\text { during } \\
\text { the first } \\
\text { postoperative } \\
\mathbf{2 4 h}\end{array}$ & $\begin{array}{c}\text { Group I: } \\
\text { IV } \\
\mathbf{( N = 5 0 )}\end{array}$ & $\begin{array}{c}\text { Group II: } \\
\text { Intraperitoneal } \\
\text { (N=50) }\end{array}$ & t-test & p-value \\
\hline Mean \pm SD & $2.34 \pm 1.08$ & $1.26 \pm 0.78$ & 32.907 & $<0.001 * *$ \\
\hline Range & $0-5$ & $0-3$ & \\
\hline
\end{tabular}

t- Independent Sample t-test; **p-value $<0.001$ HS

This table evidences highly statistically significant difference between the groups according to total analgesic equipment during the first postoperative $24 \mathrm{~h}$.

Table (14): Comparison between the groups according to nausea and vomiting

\begin{tabular}{|l|c|c|c|c|}
\hline $\begin{array}{c}\text { Nausea } \\
\text { and } \\
\text { vomiting: }\end{array}$ & $\begin{array}{c}\text { Group I: } \\
\text { IV (N=50) }\end{array}$ & $\begin{array}{c}\text { Group II: } \\
\text { Intraperitoneal } \\
\text { (N=50) }\end{array}$ & x2 & p-value \\
\hline 1st hr & $18(36.0 \%)$ & $41(82.0 \%)$ & & \\
0 & $19(38.0 \%)$ & $2(4.0 \%)$ & 24.978 & $<0.001 * *$ \\
1 & $11(22.0 \%)$ & $5(10.0 \%)$ & & \\
2 & $2(4.0 \%)$ & $2(4.0 \%)$ & & \\
3 & $45(90.0 \%)$ & $43(86.0 \%)$ & & \\
\hline 2nd hr & $3(6.0 \%)$ & $5(10.0 \%)$ & 1.879 & 0.598 \\
0 & $1(2.0 \%)$ & $2(4.0 \%)$ & & \\
1 & $1(2.0 \%)$ & $0(0.0 \%)$ & & \\
2 & $33(66.0 \%)$ & $44(88.0 \%)$ & & \\
3 & $3(6.0 \%)$ & $4(8.0 \%)$ & 10.730 & $0.013 *$ \\
\hline 3rd hr & $8(16.0 \%)$ & $1(2.0 \%)$ & & \\
0 & $6(12.0 \%)$ & $1(2.0 \%)$ & & \\
1 & & & & \\
2 & $22(44.0 \%)$ & $37(74.0 \%)$ & & \\
3 & $9(18.0 \%)$ & $7(14.0 \%)$ & 10.930 & $0.012 *$ \\
\hline 6th hr & $11(22.0 \%)$ & $4(8.0 \%)$ & & \\
0 & $8(16.0 \%)$ & $2(4.0 \%)$ & & \\
1 & & & & \\
2 & $49(98.0 \%)$ & $50(100.0 \%)$ & 1.010 & 0.315 \\
3 & $1(2.0 \%)$ & $0(0.0 \%)$ & & \\
\hline $\mathbf{2 4 t h ~ h r}$ & $0(0 \%)$ & $0(0 \%)$ & & \\
0 & $0(0 \%)$ & $0(0 \%)$ & & \\
1 & 2 & & & \\
3 & & & & \\
\hline
\end{tabular}

$\mathrm{x}^{2}$ : Chi-square test; *p-value $<0.05 \mathrm{~S} ; * *$ p-value $<0.001 \mathrm{HS} \&$ p-value $>0.05 \mathrm{NS}$

This table shows statistically significant difference between groups according to nausea and vomiting. 
Table (15): Comparison between groups according to sedation

\begin{tabular}{|l|c|c|c|c|}
\hline Sedation: & $\begin{array}{c}\text { Group I: } \\
\text { IV (N=50) }\end{array}$ & $\begin{array}{c}\text { Group II: } \\
\text { Intraperitoneal } \\
\text { (N=50) }\end{array}$ & $\mathbf{x 2}$ & p-value \\
\hline 1st hr & $2(4.0 \%)$ & $45(90.0 \%)$ & & \\
0 & $11(22.0 \%)$ & $4(8.0 \%)$ & 76.721 & $<0.001^{* *}$ \\
1 & $34(68.0 \%)$ & $1(2.0 \%)$ & & \\
2 & $3(6.0 \%)$ & $0(0.0 \%)$ & & \\
3 & $38(76.0 \%)$ & $50(100.0 \%)$ & & \\
\hline 2nd hr & $0(0.0 \%)$ & 13.636 & $<0.001^{* *}$ \\
0 & $10(20.0 \%)$ & $0(0.0 \%)$ & & \\
1 & $2(4.0 \%)$ & $0(0 \%)$ & & \\
2 & $0(0 \%)$ & & & \\
3 & $49(98.0 \%)$ & $50(100.0 \%)$ & 1.010 & 0.315 \\
\hline 3rd hr & $1(2.0 \%)$ & $0(0.0 \%)$ & & \\
0 & $0(0 \%)$ & $0(0 \%)$ & & \\
1 & $0(0 \%)$ & $0(0 \%)$ & & \\
2 & $49(98.0 \%)$ & $50(100.0 \%)$ & 1.010 & 0.315 \\
3 & $1(2.0 \%)$ & $0(0.0 \%)$ & & \\
\hline 6th hr & $0(0 \%)$ & $0(0 \%)$ & & \\
0 & $0(0 \%)$ & $0(0 \%)$ & & \\
1 & & & & \\
2 & $50(100 \%)$ & $50(100 \%)$ & 0.000 & 1.000 \\
3 & $0(0 \%)$ & $0(0 \%)$ & & \\
\hline $\mathbf{2 4 t h ~ h r}$ & $0(0 \%)$ & $0(0 \%)$ & & \\
0 & $0(0 \%)$ & $0(0 \%)$ & & \\
1 & 2 & & & \\
3 & & & & \\
\hline
\end{tabular}

$\mathrm{x}^{2}$ : Chi-square test; $*$ p-value $<0.001 \mathrm{HS} \& \mathrm{p}$-value $>0.05 \mathrm{NS}$

This table uncovers highly statistically significant difference between groups according to sedation $1^{\text {st }} \mathrm{hr}$ and $2^{\text {nd }} \mathrm{hr}$.

\section{DISCUSSION}

Pain is a consistent and predominant complaint of most individuals following surgical interventions. Failure to relieve pain is morally and ethically unacceptable. Adequate pain relief could be considered a basic human right ${ }^{(8)}$. Because of, outpatient surgery has become increasingly important in reducing the waiting lists and health care costs, especially in poor countries. Therefore, laparoscopic procedures have become very popular in recent years because of the proven quicker postoperative recovery, low rates of early and late postoperative complications, early mobilization and discharge home ${ }^{(9)}$. Pre-emptive analgesia has gained popularity and its role in the control of pain has been extensively studied in this context It involves administration of analgesic regime before the onset of noxious stimulus, with the goal of preventing sensitization of C.N.S to subsequent stimuli that amplify pain. The effect of magnesium on perioperative analgesic requirements was first evaluated by Koinig and colleagues in patients with identical levels of surgical stimulation ${ }^{(10)}$. This study was carried out on 100 adult patients of both genders and they were categorized into two groups (50 each): Group 1: Intravenous group: (IV group): $50 \mathrm{mg} / \mathrm{kg}$ magnesium sulphate in 250 $\mathrm{ml}$ of isotonic $0.9 \%$ N.S infused intravenously over 30 minutes with the beginning of operation. Group 2: Intraperitoneal group: (IP group): $50 \mathrm{mg} / \mathrm{kg}$ Magnesium sulphate in $30 \mathrm{ml}$ of isotonic $0.9 \%$ N.S installed intra peritoneal At the end of surgery. Our study showed that intravenous infusion of Magnesium sulphate attenuates arterial pressure increase and stress response more than intraperitoneal instillation during laparoscopic cholecystectomy which appears obviously at time of extubation (tex) as systolic blood pressure was (77.10 \pm 8.68 and $84.42 \pm 7.62$ respectively) while heart rate was $(92.14 \pm 12.29$ and $96.28 \pm 13.31$ respectively). The results of Jee et al. ${ }^{(3)}$, agree with our findings as they administered magnesium sulphate $\left(\mathrm{MgSO}_{4}\right) 50 \mathrm{mg} / \mathrm{kg}$ intravenously before pneumoperitoneum in patients undergoing laparoscopic cholecysyectomy and found that a close relationship exists between increases in plasma levels of catecholamines and vasopressin and arterial pressure during pneumoperitoneum. Furthermore, the administration of magnesium sulphate before pneumoperitoneum effectively attenuated arterial pressure increases in subjects undergoing laparoscopic cholecystectomy ${ }^{(3)}$. There was no bradycardia associated with the bolus magnesium given IV group after its administration immediately or later on. The efficacy of intraperitoneal instillation of local anesthetics, alone or in combination with other drugs, has been demonstrated in numerous studies on laparoscopic cholecystectomy, but there is no consensus regarding the dose, concentration, site, and manner of administration. Intraperitoneal local anesthetics block the visceral afferent signaling, potentially modifying visceral nociception and providing analgesia. Also, absorption of local anesthetics from a large peritoneal surface may be a further mechanism of analgesia (11). Ali et al. (12), concluded that intraperitoneal instillation of magnesium sulphate $\left(\mathrm{MgSO}_{4}\right)$ attenuated the hemodynamic stress response to 
pneumoperitoneum, as well as reduced postoperative pain, nausea, and vomiting in patients undergoing laparoscopic cholecystectomy (12). Magnesium decreases calcium influx to the cell, and also antagonizes NMDA receptors, which have an important role in neuronal signaling and pain processing in the central nervous system. By blocking this receptor, magnesium sulphate $\left(\mathrm{MgSO}_{4}\right)$ decreases postoperative pain due to blockage of both somatic and visceral pain fibers (11). In these studies magnesium sulphate $\left(\mathrm{MgSO}_{4}\right)$ improved the quality of analgesia, with fewer requirements for postoperative analgesics. And improve postoperative pain after laparoscopic cholecystectomy. In the present study, as regard to incisional pain the post-operative pain was assessed using visual analogue scale (VAS) and it was found that IP group have less VAS score than IV group were IV group had significantly higher scores in the 1 st, $2^{\text {nd }}$ and $3^{\text {rd }}$ hours postoperatively, while in IP group there was significantly higher scores at the 6th hour postoperative. As regard to intra-abdominal pain it was found that IP group have less VAS score than IV group were IV group had significantly higher scores in the $1 \mathrm{st}, 2^{\text {nd }}, 3^{\text {rd }}$ and $6^{\text {th }}$ hours postoperatively. Concerning shoulder pain it was found that IP group have less VAS score than IV group were IV group had significantly higher scores in the $1 \mathrm{st}, 2^{\text {nd }}, 3^{\text {rd }}, 6^{\text {th }}$ and 24 hours postoperatively. In agreement with the present results, Tramer and Glynn ${ }^{(13)}$ found that there was a similar analgesic effect of magnesium in patients undergoing elective abdominal surgery ${ }^{(13)}$. Anjum et al. (10), study showed that Magnesium sulphate $50 \mathrm{mg} / \mathrm{kg}$ in 250 $\mathrm{ml}$ of isotonic $0.9 \%$ sodium chloride solution administered intravenously over 15 to 20 minutes in the preoperative room solution alleviate postoperative pain throughout the first day after laparoscopic cholecystectomy under balanced general anesthesia significantly and reduce opioid consumption as well. Which agreed with our study as regard IV group (group 1) ${ }^{(10)}$. Saadawy et al. (14), reported that IV lidocaine and magnesium improved post-operative analgesia and reduced intraoperative and post-operative opioid requirements in patients undergoing laparoscopic cholecystectomy (14). Abdel Rouf and Amer (15), studied the postoperative analgesic effects of intraperitoneal NMDA receptor antagonist, magnesium sulphate and ketamine in patients undergoing laparoscopic cholecystectomy. They used $30 \mathrm{mg} / \mathrm{kg}$ of magnesium sulphate in patients receiving intraperitoneal $0.25 \%$ bupivacaine and 1 $\mathrm{mg} / \mathrm{kg}$ of ketamine along with $0.25 \%$ bupivacaine and concluded that demand for first analgesia in NMDA receptor antagonist was around 130 minutes after surgery ${ }^{(15)}$. Maharjan and Shrestha (16), investigations also showed 2-5 hours of less pain period in patients with intraperitoneal instillation of bupivacaine and magnesium sulphate. This conclusions is simillar to our results as regard intraperitoneal group which was 3-5 hours which mean that our dose was more effective than using magnesium sulphate $30 \mathrm{mg} / \mathrm{kg}$ plus bupivacaine. On the other hand when we used magnesium sulphate only we can avoid complications of wrong injection of bupivacaine and avoid its toxicity ${ }^{(16)}$. As regards the time for first request of analgesia (Diclofenic sodium), it was found that in IP group it was significantly longer than IV group demonstrating that the intraperitoneal magnesium sulphate prolonged the time for first demand of analgesia and the intraperitoneal magnesium is more effective than the intravenous. As for total analgesic consumption either NSAID or opioids it was found that IP group significantly consumed less analgesics than IV group which means that NMDA receptor antagonists decreased the consumption of postoperative analgesics. Magnesium sulphate had been used in several studies without any side effects in accordance with our results such as Mentes et al. (1), who reported no evidence of adverse effect owing to magnesium sulphate, Recovery and postoperative analgesia in laparoscopic cholecystectomy ${ }^{(1)}$. Shoebi et al. ${ }^{(17)}$, showed that the usage of Magnesium sulphate as adjunct, parenterally or intraperitoneally improves analgesic efficacy in postoperative period without any unwanted effects ${ }^{(17)}$. Osamu et al. ${ }^{(18)}$, injected NMDA receptor antagonists (Mg and Ketamine) into the skin of the medial region of forearm of healthy volunteers to investigate whether these substances are capable of changing the sensory transmission, and he found that intracutaneous injections of $\mathrm{Mg}$ and Ketamine cause hypesthesia to noxious and innoxious mechanical stimulations (18). Patients in IV group required more time to reach an Alderete score of 9 to be discharged from the PACU. Also having higher sedation score in the $1^{\text {st }} 2$ hours postoperative than IP group. In 
accordance to our results Ali et al. ${ }^{(12)}$, that used two groups: group $\mathrm{M}$ and group $\mathrm{C}$. Patients in group $\mathrm{M}$ received $20 \mathrm{ml}$ of $\mathrm{MgSO} 410 \%$ instilled intraperitoneally after pneumoperitoneum was created before any dissection, whereas group $\mathrm{C}$ patients received the same volume of $0.9 \%$ sodium chloride. They showed results that sedation was significantly higher in group $\mathrm{M}$ when compared with group $\mathrm{C}$ during the first $3 \mathrm{~h}$. Patients required more time to reach an Alderete score of 9 to be discharged from the PACU ${ }^{(\mathbf{1 2})}$. In our study we used magnesium sulphate $50 \mathrm{mg} / \mathrm{kg}$ which was safe as we did not observe any signs of magnesium toxicity. Magnesium sulphate had been used in several studies without any side effects in accordance with our results such as Mentes et al. (1), who reported no evidence of adverse effect owing to magnesium sulphate was reported. Recovery and postoperative analgesia in laparoscopic cholecystectomy as well as in thoracotomies have shown favorable results using magnesium sulphate ${ }^{(\mathbf{1})}$.

\section{CONCLUSION}

Laparoscopic cholecystectomy definitely results in less post-operative pain than its open counterpart but it isn't a pain free procedure. Pain after laparoscopic cholecystectomy can be subdivided into viscenil, parietal and shoulder pain. They have different intensities and their own time courses. The intraperitoneal route of administration magnesium sulphate at the end of laparoscopic cholecystectomy is a simple technique and without complications. It provides early post-operative pain relief that is particularly suitable for the practice of ambulatory anesthesia. NMDA receptor antagonists (Magnesium sulphate) augmented the post-operative analgesia, reduced the post-operative pain, prolonged the time for the first need ofanalgesia and shortened the hospital stay, whether used intraperitoneal or intravenous. The presence of peripheral (peritoneal) NMDA receptors plays an important role in normal visceral pain transmission and it provides a novel mechanism for development of peripheral sensitization and visceral hyperalgesia. Intraperitoneal magnesium sulphate was more effective than intravenous magnesium sulphate. The central and peripheral NMDA receptors should be blocked for the best postoperative analgesia which was achieved by the intraperitoneal magnesium sulphate on the contrary to the intravenous which only blocked the central receptors. Finally: The intraperitoneal administration of magnesium sulphate is a safe and effective method in the management of acute postoperative pain after laparoscopic cholecystectomy more than intravenous administration of magnesium sulphate.

\section{CONFLICTS OF INTEREST}

There are no conflicts of interest.

\section{REFERENCES}

1. Mentes O, Harlak A, Yigit T et al. (2008): Effect of intraoperative magnesium sulphate infusion on pain relief after laparoscopic cholecystectomy. Acta Anaesthesiol Scand., 52: 1353-1359.

2. Fredheim OM, Borchgrevink $P C$ and Kvarstein G (2011): Post-operative pain management in hospitals. Tidsskr Nor Laegeforen, 131(18): 1772e6.

3. Jee D, Lee D, Yun S and Lee C (2009): Magnesium sulphate attenuates arterial pressure increase during laparoscopic cholecystectomy. British Journal of Anaesthesia, 103 (4): 484-489.

4. Sung JH, Eun JC, Ji YL et al. (2003): The physiologic respons to laparoscopic cholecystectomy: $\mathrm{CO} 2$ pneumoperitoneum vs wall lift. Can J Anaesth., 50: 200-1.

5. Shah PN and Dhengle $Y$ (2016): Magnesium sulfate for postoperative analgesia after surgery under spinal anesthesia. Acta Anaesthesiologica Taiwanica, 54 62-64.

6. Enes H, Semir I, Šefik H et al. (2011): Postoperative pain in open vs. laparoscopic cholecystectomy with and without local application of anaesthetic. Medicinski Glasnik, 8(2):243-247

7. Olivieri L and Plourde G (2005): Prolonged (more than ten hours) neuromuscular blockade after cardiac surgery: Report of two cases. Can J Anaesth., 52: 88-93.

8. Tashi C, Nongthombam RS, Langpolakpam CS et al. (2014): The effect of pregabalin for relief of postoperative pain after abdominal hysterectomy, Journal of Medical Society, 28(1):18-21 
9. Weinbroum AA (2003): A single small dose of postoperative ketamine provides rapid and sustained improvement in morphine analgesia in - the presence of morphine- resistant pain. Anesth. Analg.,96: 789795.

10. Anjum S, Waism $S$ and Sabeeha $G$ (2015): Effect of Magnesium Sulphate on postoperative pain in laparoscopic cholecystectomy. International Journal of Advanced Research, 3(10): 813 - 818.

11. Marks JL, Ata B and Tulandi T (2012): Systematic review and Meta-analysis of intraperitoneal instillation of local anesthetics for reduction of pain after gynecologic laparoscopy. J Minim Invasive Gynecol., 19: 545-553.

12. Ali RM, Rabie AH, Elshalakany NA et al. (2015): Effect of intraperitoneal magnesium sulfate on hemodynamic changes and its analgesic and antiemetic effect in laparoscopic cholecystectomy. Ain Shams Journal of Anesthiology, 8(2): 153-159.

13. Tramer MR and Glynn CJ (2007): An evaluation of a single dose of magnesium to supplement analgesia after ambulatory surgery: randomized controlled trail. Anesth. Analg., 104: 1374-9.
14. Saadawy IM, Kaki AM, Abd El Latif AA et al. (2010): Lidocaine vs. magnesium: effect on analgesia after a laparoscopic cholecystectomy. Acta Anaesthesiol Scand, 54: 549-556.

15. Abdel-Raouf $M$ and Amer $H$ (2004): Postoperative analgesic effects of intraperitoneal NMDA receptor antagonists (ketamine and magnesium) in patients undergoing laparoscopic cholecystectomy. Engl J Anaesth., 20(2): 107-11.

16. Maharjan SK and Shrestha S (2012): Intraperitoneal magnesium sulphate plus bupivacaine for pain relief after laparoscopic cholecystectomy. Journal of Kathmandu Medical College, 1(1): 1-7.

17. Shoebi G, Abolfazl $F$ and Farzaneh $T$ (2007): The additional effect of magnesium sulfate to lidocaine in spinal anesthesia for cesarean section. Int $\mathbf{J}$ Pharmacol., 3(5): 425-7

18. Osamu I, Takahiro U and Toshikazu T (2002): Peripheral Administration of Magnesium Sulfate and Ketamine Hydrochloride Produces Hypesthesia to Mechanical Stimuli in Humans. J. Health Sci., 48: 69-72. 